\title{
Oscillatory Characteristics of Nociceptive Responses in the SII Cortex
}

\author{
Fu-Jung Hsiao, Wei-Ta Chen, Kwong-Kum Liao, Zin-An Wu, Low-Tone Ho, \\ Yung-Yang Lin
}

\begin{abstract}
Objective: This study is aimed to explore the frequency characteristics of pain-evoked neuromagnetic responses in the secondary somatosensory (SII) cortices. Methods: Thulium-laser nociceptive stimuli to the left hand dorsum of 10 right-handed healthy adults. The pain stimuli were rated as mild, moderate, and severe levels according to subjects' reports on a 10-point visual analog scale. We analyzed their cortical responses with wavelet-based frequency analyses and equivalent current dipole (ECD) modeling. Results: For each pain level, we found an increase of theta $(4-8 \mathrm{~Hz})$ and alpha $(8-13 \mathrm{~Hz})$ power in bilateral SII areas at $180-210 \mathrm{~ms}$ after stimulus onset. The power was larger for the moderate than for the mild pain level $(\mathrm{p}<0.05)$, but there was no statistical power difference of these oscillations between moderate and severe pain stimulus conditions $(\mathrm{p}=0.7)$. Within the SII area, we did not observe particular difference in theta and alpha ECD locations between varying pain level conditions. Conclusions: The 4-13 Hz activities, peaking from 180 to $210 \mathrm{~ms}$, are oscillatory correlates of SII activation in response to nociceptive stimulation, but their power may code the magnitude of pain stimuli only up to moderate level, as rated subjectively. This measure could be potentially used to evaluate SII activation in further pain studies.
\end{abstract}

RÉSUMÉ: Caractéristiques oscillatoires des réponses nociceptives dans le cortex SII. Objectif : Le but de cette étude était d'explorer les caractéristiques des fréquences des réponses neuromagnétiques évoquées par la douleur dans les cortex somatosensitifs secondaires (SII). Méthodes : Des stimuli nociceptifs au laser-thulium ont été appliqués à la face dorsale de la main gauche de 10 adultes droitiers en bonne santé. Les stimuli douloureux étaient évalués comme étant légers, modérés ou sévères par les sujets au moyen d'une échelle analogue visuelle de 10 points. Nous avons analysé leurs réponses corticales au moyen d'analyses fréquentielles par ondelettes et de modélisation d'un dipôle de courant équivalent (DCÉ). Résultats : Pour chaque niveau de douleur, nous avons observé une augmentation de puissance thêta (4-8 Hz) et alpha (8-13 Hz) dans les aires SI bilatérales, 180 à $210 \mathrm{~ms}$ après le début du stimulus. La puissance était plus grande lors de la douleur modérée par rapport à la douleur légère ( $\mathrm{p}<0,05)$, mais il n'y avait pas de différence statistique dans la puissance de ces oscillations entre la douleur modérée ou sévère ( $p=0,7)$. Nous n'avons pas observé de différence particulière dans la localisation DCÉ thêta et alpha dans la zone SII selon les niveaux de douleur. Conclusions : Les activités 4$13 \mathrm{~Hz}$, dont le pic était observé entre 180 et $210 \mathrm{~ms}$, sont des corrélats oscillatoires de l'activation au niveau du SII en réponse à une stimulation nociceptive, mais leur puissance peut témoigner de l'ampleur de stimuli douloureux seulement jusqu'à un niveau modéré, évalué subjectivement. Cette mesure pourrait potentiellement être utilisée pour évaluer l'activation SII dans des études ultérieures sur la douleur.

Can. J. Neurol. Sci. 2008; 35: 630-637

Electrophysiological studies on pain-evoked neuronal responses are crucial for a better understanding of pain perception mechanisms in humans. Noxious stimulation with short-pulse laser was introduced to pain research around three decades ago. ${ }^{1}$ Unlike electric stimulation, laser stimulation selectively activates cutaneous nociceptive receptors without simultaneously eliciting a tactile response. ${ }^{2}$ Thus the peripheral A-delta $(\mathrm{A} \delta)$ and $\mathrm{C}$ fibers are involved in the generation of the resultant laser-evoked potential (LEP) and magnetic field (LEF) recorded by electroencephalography (EEG) and magnetoencephalography (MEG), respectively., ${ }^{1,3-5}$ With a better spatial resolution, MEG is more suitable than EEG for studying pain processing. ${ }^{4,6-8}$ Previous time-domain analyses of pain-elicited brain responses have shown the involvement of complex cortical networks including the primary (SI) and secondary somatosensory (SII) cortices, the insular cortex, the anterior cingulate cortex, and the dorsolateral prefrontal cortex.9-12 However, the frequency characteristics of pain-evoked responses remain unclear. ${ }^{13}$

The information obtained with either EEG or MEG reflects an ensemble of neuronal sources that generate oscillatory

From the Institute of Physiology (FJH, LTH, YYL), Institute of Brain Science (FJH, WTC, YYL), Institute of Neuroscience (WTC,), Institute of Clinical Medicine (YYL), Department of Neurology (KKL, ZAW), National Yang-Ming University; Neurological Institute (WTC, KKL, ZAW), Department of Medical Research and Education (FJH, LTH, YYL), Taipei Veterans General Hospital, Taipei, Taiwan. Received January 3, 2008. Final Revisions Submitted April 22, 2008. Correspondence to: Yung-Yang Lin, Department of Medical Research and Education, and Department of Neurology, Taipei Veterans General Hospital, No.201, Sec.2, Shih-Pai Rd., Taipei 112, Taiwan. 
activities in various frequency ranges. Responding to adequate peripheral stimulation, these sources are coherently activated and coupled with resultant change in brain rhythms. ${ }^{14,15}$ Previous studies have reported the participation of delta to gamma bands in the visual and auditory evoked activations. ${ }^{14-22}$ Recent EEG studies have shown frequency-specific over-activations in patients with neurogenic pain, which suggests a role of the thalamocortical loop in pain processing. ${ }^{23,24}$ Moreover, Hauck and coworkers have reported an involvement of high-frequency activity in the cerebral mechanisms of attentional augmentation of pain processing. ${ }^{25}$ Therefore, it is worthy further investigating the oscillatory dynamics of pain-evoked brain responses.

Most biosignals that vary around a mean value can be reconstructed as a sum of sine and cosine waves occurring at different frequencies. ${ }^{26}$ The spectral wavelet analysis allows the overall variance of a biosignal to be split into individual frequency components. ${ }^{26}$ Power difference between various oscillations might be attributed to differential involvement in pain processing. We therefore hypothesized that some oscillations might specifically reflect the correlation between SII activation and subjective pain rating. In this study, we identified the oscillatory components of laser-evoked MEG responses by using wavelet transform analysis, and then compared with their power. To explore this hypothesis, this study involves: a) documenting the quantitative changes of pain-related oscillatory activities, b) analyzing the correlations between perceived pain magnitude and the oscillatory activities of the SII cortex, and c) tracking the cortical representations of pain-related oscillatory activities.

\section{Methods}

\section{Subjects}

Ten healthy volunteers (eight men and two women; mean age $32.1 \pm 4.3$ years; all right-handed) were recruited to participate in this study. None had any neurological or psychiatric deficits. Each subject gave their informed consent prior to the experiment. Our study protocol was approved by the institutional review board of Taipei Veterans General Hospital.

\section{Laser pulse stimulation and pain rating}

Cutaneous nociceptive stimuli were produced using a thulium-YAG laser stimulator (BLM 1000 Tm:YAG®, Baasel Lasertech, Starnberg, Germany) set up in the MEG lab at Taipei Veterans General Hospital. The laser emits near-infrared radiation with a wavelength of $1.96 \mu \mathrm{m}$, a spot area of $10 \mathrm{~mm}^{2}$, and a pulse duration of $1 \mathrm{~ms}$; resulting in a penetration depth of $360 \mu \mathrm{m}$ into the human skin. This laser beam was then conducted via an optical fiber, into a magnetically shielded room through a small hole. The stimuli were applied to the lateral dorsum of the left hands of the volunteers by an assistant who held the handpiece at the end of the optical fibre and kept the stimulator head stably placed on the skin. In order to avoid skin burns and fatigue of the primary nociceptive afferents, our research assistant slightly changed the position of hand piece within an area of 3-4 $\mathrm{cm}$ in diameter following each stimulus. To find the three different laser pulse intensities rated by each subject as mild, moderate and severe pain respectively, we asked all the subjects to rate a train of laser pulse stimulations starting from $100 \mathrm{~mJ}$ and increasing in $50 \mathrm{~mJ}$ steps. Each subject was instructed to rate the perceived intensity of a stabbing pain using the Visual Analogue Scale (VAS). ${ }^{27,28}$ We determined pain threshold as the lowest intensity level that evoked clear stabbing pain (VAS $=1$ ). The VAS 0 was defined as no pain, and VAS 10 as the worst imaginable pain. We determined the lowest strengths of laser pulses for eliciting pain levels at VAS 2-3, VAS 5-6, VAS 8-9 for each subject, and then applied the stimuli on each subject to elicit mild, moderate and severe pain, respectively. The above methodology for stimulation and pain rating has been detailed elsewhere. ${ }^{6}$ Accordingly, the stimulus intensities for producing mild, moderate, and severe pain were determined to be 255,365 , and $490 \mathrm{~mJ}$, respectively, when averaged across all subjects.

\section{MEG measurement}

During the MEG recordings, each subject sat comfortably in a magnetically shielded room with the head supported against the helmet-shaped bottom of a whole-scalp 306-channel neuromagnetometer (Vectorview ${ }^{\mathrm{TM}}$, Elekta Neuromag, Helsinki, Finland). Our neuromagnetometer comprised 102 identical triple sensor elements, and each sensor element consisted of one magnetometer and two orthogonal planar gradiometers. In the present study, the data analysis was based on the signals of the 204 planar gradiometers, because of relatively poor signal-tonoise ratio for magnetometer signals. ${ }^{29}$

Each subject underwent three sessions (mild, moderate, and severe pain) of laser pulse stimulation in a randomized order. Before each session, the subject had a five to ten minute rest. Forty responses were averaged in each session. The interstimulus interval (ISI) varied between 8 and $12 \mathrm{~s}$. The signals were bandpass filtered $(0.1-160 \mathrm{~Hz})$ and digitized at $500 \mathrm{~Hz}$. Epochs were excluded from being averaged whenever the amplitudes of the corresponding electro-oculogram and MEG signals were larger than $300 \mu \mathrm{V}$ and $6000 \mathrm{fT} / \mathrm{cm}$, respectively.

The exact location of the head with respect to the sensors was found by measuring magnetic signals produced by currents that were led to four head indicator coils, placed at known sites on the scalp. The locations of the coils with respect to anatomical landmarks on the head were determined with a threedimensional (3-D) digitizer to allow alignment of the MEG and magnetic resonance (MR) image coordinate systems. ${ }^{29}$ The MR images of the brain of each of the subjects were acquired with a 3 T Bruker Medspec300 scanner (Germany).

\section{Wavelet analyses and equivalent current dipole (ECD) modeling}

The LEF responses of the gradiometer channels were computed with the continuous wavelet transform by using MATLAB 6.5 programming software (The MathWorks, Natick, MA, USA). The analysis period of $1100 \mathrm{~ms}$ included a prestimulus baseline of $100 \mathrm{~ms}$. The Morlet wavelet ${ }^{30}$ is a function of time $t$ and frequency $f_{0}$ defined as:

$$
\begin{gathered}
\mathrm{w}\left(\mathrm{t}, \mathrm{f}_{0}\right)=A \exp \left(-\mathrm{t}^{2} /\left(2 \sigma_{\mathrm{t}}^{2}\right)\right) \exp \left(\mathrm{i} 2 \pi \mathrm{f}_{0} \mathrm{t}\right), \\
\text { where } \sigma_{\mathrm{t}}=1 /\left(2 \pi \sigma_{\mathrm{f}}\right) \text { and } A=1 /\left(2 \pi \sigma_{\mathrm{t}}^{2}\right)^{1 / 2}
\end{gathered}
$$

The width of the wavelet $\left(\mathrm{m}=\mathrm{f}_{0} / \sigma_{\mathrm{f}}\right)$ was chosen to be $7 .{ }^{31-38}$ The time-varying amplitude of the neuromagnetic responses in a frequency band around $\mathrm{f}_{0}$ is the result of the convolution of the complex wavelet $\mathrm{w}\left(\mathrm{t}, \mathrm{f}_{0}\right)$ with the signal $\mathrm{s}(\mathrm{t})$ : 


$$
\mathrm{E}\left(\mathrm{t}, \mathrm{f}_{0}\right)=\mathrm{w}\left(\mathrm{t}, \mathrm{f}_{0}\right) * \mathrm{~s}(\mathrm{t})
$$

This procedure was performed by using a set of wavelets with $\mathrm{f}_{0}$ ranging from 0.5 to $25 \mathrm{~Hz}$ at intervals of $0.5 \mathrm{~Hz}$.

Time-frequency representation of pain-related responses was obtained from the squared norm of $\mathrm{E}\left(\mathrm{t}, \mathrm{f}_{0}\right)$ with $\mathrm{f}_{0}$ ranging from 0.5 to $25 \mathrm{~Hz}$ in all channels. The spatial distribution, power and temporal features of the stimulus-related oscillatory activities were exhibited. To see the oscillatory characteristics following laser stimulation, we inspected the pain-related time-frequency representations, and selected the single channel with maximal oscillatory activities located around the SII from both hemispheres for further analysis. The time-frequency plots for the selected channels were averaged across individual frequency bands of $0.5-4 \mathrm{~Hz}, 4-8 \mathrm{~Hz}, 8-13 \mathrm{~Hz}$ and $13-25 \mathrm{~Hz}$ to provide the time-varying measures of delta, theta, alpha and beta activities, respectively. The mean power value during the $100 \mathrm{~ms}$ prior to stimulus onset was considered as the baseline level and was subtracted from the power after the stimulus onset. Peak latencies were derived from the time point of maximal power for individual rhythmic activities.

We further averaged the $\mathrm{E}\left(\mathrm{t}, \mathrm{f}_{0}\right)$ of all the channels that related to the bands of interest mentioned above and obtained the amplitude fluctuations of the rhythmic activities. The oscillatory activities were then modeled with ECD modeling. ${ }^{29}$ To obtain the activation areas, we visually searched the oscillatory deflections that clearly exceeded the prestimulus background level. The single ECD that best described the measured data was found by a least-squares search using the subsets of 24-30 channels around the maximal responses. Goodness-of-fit of the model was calculated and only ECDs explaining more than $80 \%$ of the field variance at selected periods of time over the subset of channels were used for further analysis. These calculations resulted in the 3-D location and orientation of the ECD in a spherical conductor, which were coregistered with the MR images of each subject's brain. The positive $\mathrm{x}^{-}, \mathrm{y}^{-}$, and $\mathrm{z}$-axes in our head-coordinate system were set towards the right preauricular point, the nasion, and the head vertex, respectively.

\section{Statistics}

In the present study, we evaluated the peak powers, latencies and ECD locations with respect to the effects of pain intensity (mild, moderate and severe), frequency bands (delta, theta, alpha and beta), and the hemisphere (contra- and ipsi-lateral SII) by using non-parametric repeated measures ANOVA (Friedman ANOVA). When a significant effect was found, post-hoc comparisons were performed by using Wilcoxon's signed ranks test. The significance threshold was taken as $p<0.05$.

\section{RESUlts}

\section{Time-frequency representations}

Figure 1(A) shows the time-frequency representation of the evoked neuromagnetic responses of Subject 1 by moderate painful stimulation. It shows the spatial distribution of $0.5-25 \mathrm{~Hz}$ activities $100 \mathrm{~ms}$ before and $1000 \mathrm{~ms}$ after stimulus onset. The enhanced oscillatory activities are clearly discernible in the bilateral temporoparietal areas. Figure 1(B) displays the timefrequency plots from the two channels of interest located around the bilateral SII of Subject 1 in response to mild, moderate, and
(A)

(B)
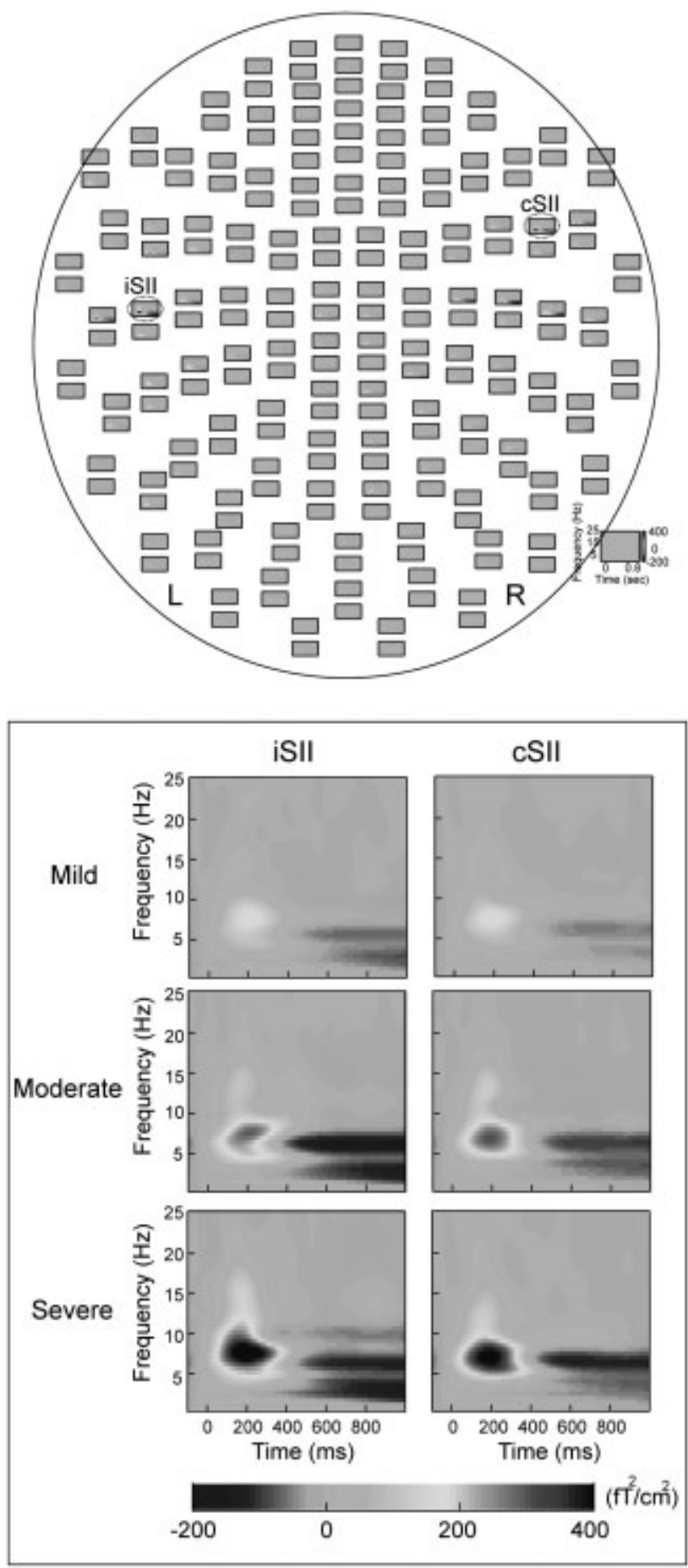

Figure 1: (A) Time-frequency representations of the neuromagnetic responses of Subject 1 to moderate pain stimulation. The head is flattened and viewed from above with the subject's nose pointing upward. Time 0 indicates the onset of stimulus, and the power relative to the 100ms pre-stimulus baseline level is coded in gray level. Clear power increase is seen over the ipsilateral (iSII) and contralateral secondary somatosensory areas (cSII). (B) Enlarged illustrations on the same channels from both hemispheres showing the time-frequency power plots in mild, moderate, and severe pain conditions. $L$, left; $R$, right. 
severe painful stimuli. A power increase was found in all conditions around $150-220 \mathrm{~ms}$ following stimulus onset, with maximal power ranging between 5 and $10 \mathrm{~Hz}$. Notably Subject 1 additionally shows long-lasting power suppression in the theta range, occurring around $500 \mathrm{~ms}$ after stimulus onset and lasting up to $1000 \mathrm{~ms}$. This finding was not, however, included in our analysis because of its inconsistency across subjects.

\section{Pain-related oscillations}

Figure 2 shows the power (mean \pm SEM) of the delta to beta frequency bands from the two channels of interest in the bilateral hemispheres in response to mild, moderate and severe pain. We observed a significant bilateral power increase in the delta, theta and alpha activities to all the three pain intensities (all $p<0.01$ ). As for the factor of frequency band, statistical analysis showed significant differences (all $\chi^{2}>12$, all $p<0.01$ ). Post hoc comparison revealed that both the theta and alpha activities in all pain intensity conditions, as well as in the bilateral hemispheres, were significantly larger than the delta and beta activities $(p<$ 0.01 ), which indicated that the pain-related oscillatory activities take place predominantly within the theta and alpha frequency ranges. With respect to the hemispheres, no significant power difference was found in any of the pain intensity conditions (all $p>0.2)$. Finally, pain intensity elicited significant differences in the theta $\left(\chi^{2}=7.8, p<0.05\right)$ and alpha $\left(\chi^{2}=9.8, p<0.01\right)$ activities in the bilateral hemispheres. Wilcoxon testing showed that the power of the theta and alpha bands under mild painful stimulation were significantly smaller than when moderate and severe pain stimuli were used (for theta, $p<0.02$; for alpha, $p<$ $0.05)$. However, bilateral theta and alpha activities were comparable in power in moderate and severe pain conditions (all $p>0.5)$.

The Table below summarizes the peak latency of delta to beta activities for different pain intensity conditions. The overall mean $( \pm$ SEM) latencies across all conditions was $194 \pm 2.4 \mathrm{~ms}$. Statistical analysis of the peak latencies showed no significant difference with respect to the frequency band $(\chi 2<5, p>0.1)$, hemisphere $(\chi 2<3, p>0.1)$, and pain intensity $(\chi 2<4, p>0.1)$.

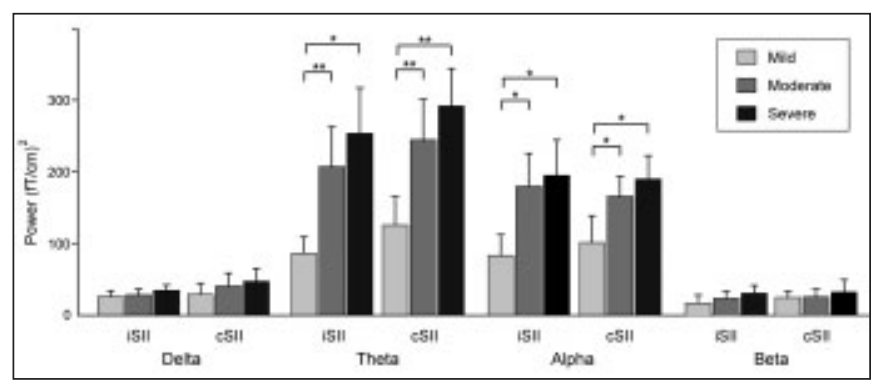

Figure 2: Mean power of delta to beta activities of iSII and cSII responses to laser stimulation with different pain intensities, obtained from the single channel with maximal oscillatory activities located around the SII in each hemisphere. $* P<0.05 ; * * P<0.01$. Delta, 0.5 $4 \mathrm{~Hz}$; Theta, 4 - $8 \mathrm{~Hz}$; Alpha, 8 - $13 \mathrm{~Hz}$; Beta, 13 - $25 \mathrm{~Hz}$.
Table: Mean ( \pm SEM) peak latency $(\mathrm{ms})$ across 10 subjects of various frequency bands in the SII ipsilateral and contralateral to pain stimuli of different pain conditions

\begin{tabular}{cccc}
\hline Pain condition & Band & Ipsilateral & Contralateral \\
\hline \multirow{4}{*}{ Mild } & delta & $209 \pm 10$ & $206 \pm 15$ \\
\cline { 2 - 4 } & theta & $210 \pm 10$ & $197 \pm 7$ \\
\cline { 2 - 4 } & alpha & $206 \pm 13$ & $189 \pm 14$ \\
\cline { 2 - 4 } & beta & $206 \pm 13$ & $189 \pm 11$ \\
\hline \hline \multirow{4}{*}{ Moderate } & delta & $209 \pm 14$ & $201 \pm 14$ \\
\cline { 2 - 4 } & theta & $187 \pm 11$ & $206 \pm 8$ \\
\cline { 2 - 4 } & alpha & $194 \pm 13$ & $182 \pm 9$ \\
\cline { 2 - 4 } Severe & beta & $182 \pm 13$ & $183 \pm 10$ \\
\hline \hline \multirow{4}{*}{} & delta & $190 \pm 13$ & $206 \pm 15$ \\
\cline { 2 - 4 } & theta & $198 \pm 8$ & $198 \pm 7$ \\
\cline { 2 - 4 } & alpha & $182 \pm 12$ & $186 \pm 9$ \\
\cline { 2 - 4 } & beta & $207 \pm 11$ & $183 \pm 10$ \\
\hline
\end{tabular}

\section{Cortical localization of pain-related oscillations}

Figures 3(A) and 3(B) show the spatial distributions of timevarying theta and alpha activities for moderate painful stimulation from Subject 1 and Subject 2, respectively. Clear response deflections are observed in the bilateral temporoparietal areas. For theta and alpha activity, one in the ipsilateral hemisphere peaks at 192 and $184 \mathrm{~ms}$; another one in the contralateral hemisphere at 186 and $182 \mathrm{~ms}$, respectively. The isocontour map suggests the presence of a single ECD in each hemisphere, which are localized in the superior bank of the Sylvian fissure, around the SII area.

Figure 4(A) shows the ECDs of theta and alpha activities of Subject 2 in various pain stimulus conditions superimposed on his own MR images. These ECDs were localized in the vicinity of the superior bank of the Sylvian fissure, corresponding to the SII cortex. Furthermore, in order to compare the ECD locations, we normalized their $\mathrm{x}^{-}, \mathrm{y}-$, and $\mathrm{z}$-coordinate values by defining those of theta activities elicited by moderate pain as 0,0 , and 0 , respectively. Figure 4(B) shows the relative values (mean \pm s.d) and distributions across the ten subjects of the theta and alpha activities for each stimulus condition. No significant difference for the factor of pain intensity was identified $\left(\chi^{2}<2, p>0.1\right)$.

\section{DiscuSSION}

In this study we used wavelet analysis and equivalent current dipole modeling to analyze the temporal and spatial characteristics of neuromagnetic oscillatory activities in bilateral SII areas following painful laser stimulation at varying pain intensities. Our first finding was the significant power increase 
(A)

Moderate Pain - Theta
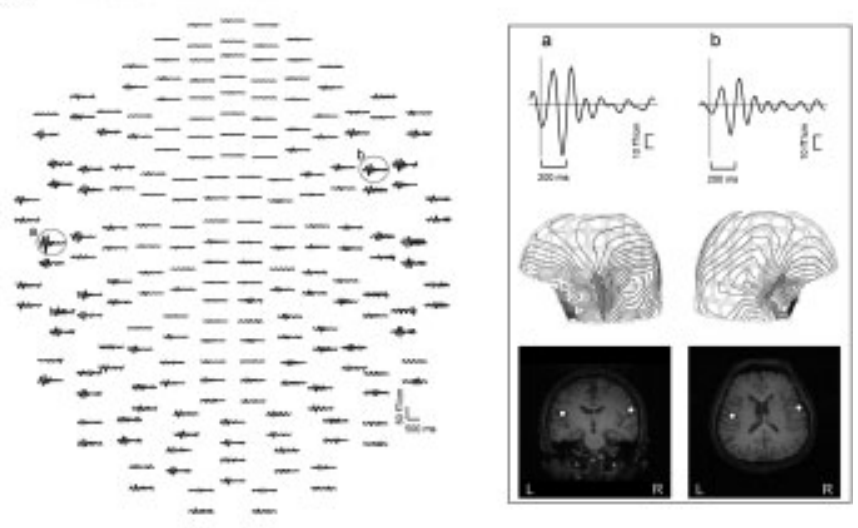

(B)

Moderate Pain - Ajpha
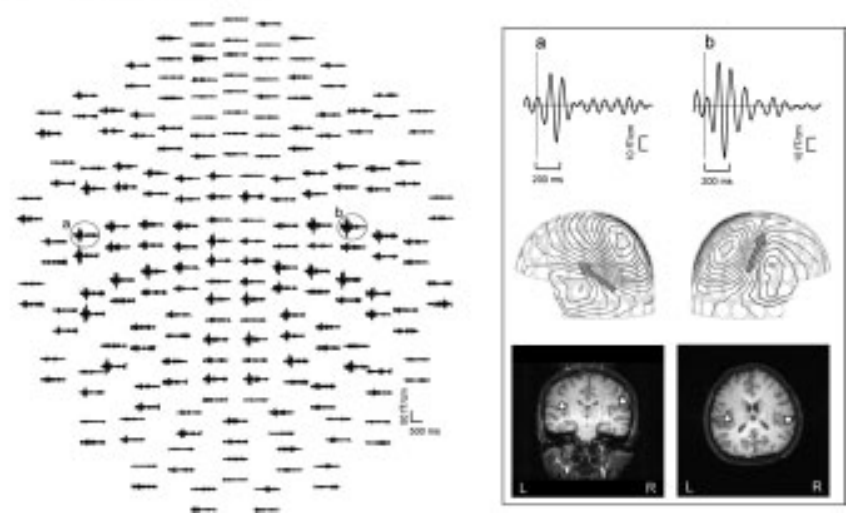

Figure 3: (A) The spatial distribution of theta activities of Subject 1 to moderate pain stimulation upon the left hand dorsum. (B) The spatial distribution of alpha activities of Subject 2 to moderate painful stimulation. The 204 recording channels are flattened and viewed from top, with the nose pointing upward. The insert shows the enlarged representative signals ( $a$ and $b$ in upper panel) from bilateral temporoparietal regions, the corresponding magnetic dipole patterns (middle panel), and source locations (white dots) on MR imaging slices (lower panel). Each arrow indicates the orientation of the corresponding equivalent current dipole. The dipole locations from theta and alpha activities were estimated in bilateral SII. L, left; $R$, right.

of delta to alpha frequency band activities in the bilateral SII areas 180 to $210 \mathrm{~ms}$ following noxious stimulation. This result is partly in line with previous EEG studies using median nerve stimulation, ${ }^{39}$ intracutaneous electrical stimulation ${ }^{40}$ and $\mathrm{CO}_{2}$ laser skin stimulation. ${ }^{41,42}$ It is also in accordance with the ideas of neuronal processing with simultaneous oscillations in various frequency bands. ${ }^{19}$ Remarkably, the salient theta and alpha activities were observed in all pain intensity conditions in the bilateral SII areas. In contrast with our findings, however, some EEG studies showed a decrease in alpha and an increase in beta oscillations following tonic thermal or chemical stimulations. ${ }^{43-}$

48 Babiloni and coworkers reported an increase of theta to
(A)

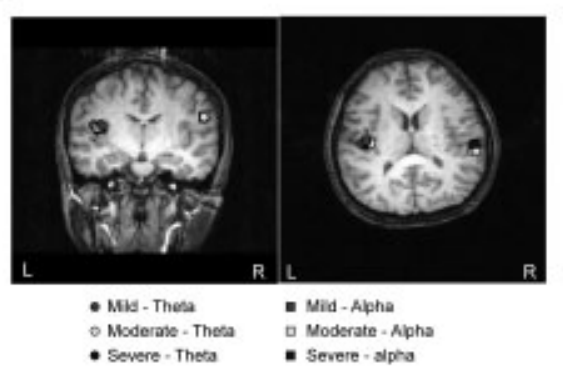

(B)

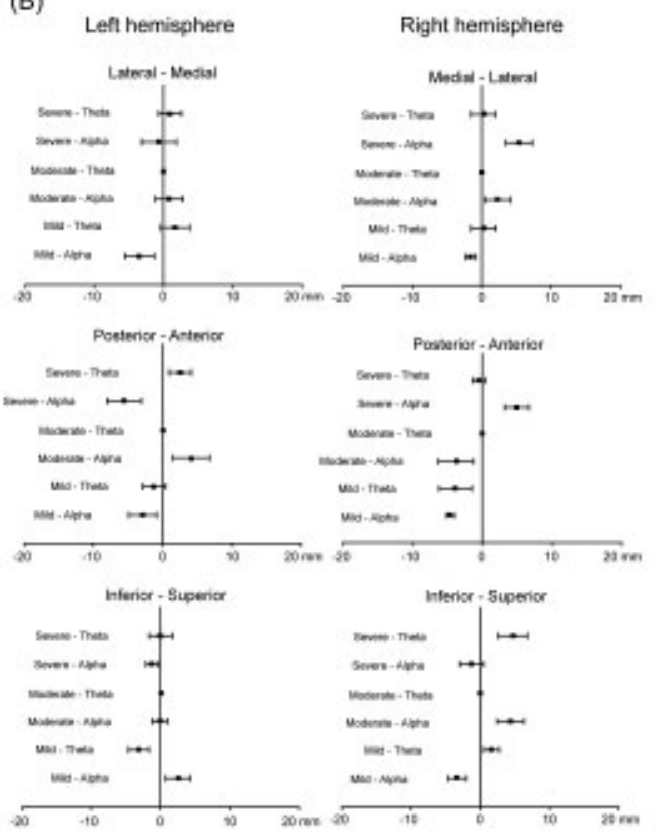

Figure 4: (A) The ECD locations of theta and alpha activities of Subject 2 in response to mild, moderate, and severe pain stimuli. (B) The relative distribution of theta and alpha ECDs in various pain conditions with respect to the locations of the theta ECD activated by moderate pain (moderate-theta) in terms of mean coordinate values (filled boxes) across ten subjects. The positive values in $x$-, $y$-, and $z$-axes suggest relatively medial, anterior, and superior distributions, respectively. The bars on each box indicate standard deviation values $( \pm$ s.d.). gamma activities in the contralateral hemisphere and a decrease of beta activity in the ipsilateral hemisphere following painful electrical stimuli. ${ }^{49}$ This divergence might be related to discordant pain-eliciting modalities and different analysis methodologies. It is noteworthy that our study has its strengths in the selective activation of nociceptive neurons ${ }^{2}$ and precise localization in time, space and frequency, in comparison with previous EEG spectral analyses which used Fourier transform. ${ }^{43-}$ ${ }^{49} \mathrm{We}$ therefore believe that the theta and alpha activities are essentially engaged in cortical pain processing. The functional significance of the cortical oscillations in the SII cortex has, however, remained somewhat unclear. One recent MEG study ${ }^{50}$ 
has reported 7-9 $\mathrm{Hz}$ rhythm in the human SII cortex, probably processing the bilateral tactile inputs. We therefore propose that theta and alpha activities in the SII areas may play an important role in processing nociceptive inputs.

In this study, the latency range of the bilateral oscillatory activities is in agreement with that reported in earlier LEP. ${ }^{51-54}$ and LEF studies. ${ }^{4,8,55-57}$ Moreover, the latency between 180 and $210 \mathrm{~ms}$, irrespective of the pain intensity and the hemispheres, may suggest that the sensory input is conducted via the ascending A f fibers. No hemispheric lateralization of nociceptive processing in SII areas was found to result from there being no significant power difference in oscillatory activities between the contralateral and ipsilateral hemispheres in all pain intensity conditions. Coghill and colleagues ${ }^{58,59}$ also suggested that pain intensity-dependent activation of the SII cortex was predominantly bilateral in their positron emission tomography (PET) studies. Moreover, psychophysical studies of patients with one cerebral hemisphere excision ${ }^{60}$ or split-brain ${ }^{61}$ have also confirmed that both cerebral hemispheres can independently process pain intensity information. Our present observation of similar oscillatory activities between the bilateral SII areas supports this idea of the bilateral mechanism being engaged in the nociceptive processing.

In this study, the power increase of theta and alpha activities, when correlated with pain intensity levels, showed a tendency to increase from a moderate to a severe condition, although this tendency was not significant. Recent studies on EEG responses to $\mathrm{CO}_{2}$ laser skin stimulation ${ }^{41}$ have suggested that event-related synchronization activities from delta to alpha bands, with a mean latency of $225 \mathrm{~ms}$, increase in positive correlation with stimulus strength from 3-11 $\mathrm{mJ} / \mathrm{mm}^{2}$. Mouraux et al have reported a change of event-related synchronization from non-painful to mild painful stimulation. ${ }^{41}$ In our study, however, a dissimilar stimulus paradigm was used as the mean stimulus strengths were calculated to be $25.5,36.5$ and $49 \mathrm{~mJ} / \mathrm{mm}^{2}$ for mild, moderate and severe pain, respectively. We found that the theta and alpha activities in the SII areas code for stimulus strengths of up to $36.5 \mathrm{~mJ} / \mathrm{mm}^{2}$ or up to a moderate pain intensity. The difference in energy densities used by Mouraux et $\mathrm{al}^{41}$ and the present study may be explained by the difference in wavelength of the laser stimulators used. As the skin has a higher transparency for the shorter wavelength of the thulium-YAG laser, as compared to the $\mathrm{CO}_{2}$ laser, much more calorific energy is needed to bring a larger volume of tissue above the activation threshold of nociceptors. Raij et $\mathrm{al}^{56}$ have shown that the amplitude of LEF responses increases strongly along with the increment of interstimulus interval (ISI) from $0.5 \mathrm{~s}$ to $4 \mathrm{~s}$, and then reaches a plateau at the ISI of 8 to $16 \mathrm{~s}$. Moreover, unlike $\mathrm{CO}_{2}$ laser stimuli, our thuliumlaser stimuli last for only $1 \mathrm{~ms}$. It seems not possible for an acclimation to the stimuli to be developed during our experiments with ISI of 8-12 s. Moreover, in our present study, the order of various experimental sessions (mild, moderate, and severe pain) was randomized, and the subject had a five-ten minute rest before each session. Thus, the lack of significant change between moderate and severe pain conditions cannot be ascribed to the acclimation effect.

In both hemispheres, the spatial distributions of theta and alpha activities to nociceptive information processing closely resembled each other in all pain-intensity conditions. The oscillations were centered in the SII region in the bilateral hemispheres, in accordance with previous scalp EEG, ${ }^{51-54,62}$ intracranial EEG, ${ }^{63-67}$ and MEG recordings. ${ }^{4,5,8,57,68-70} \mathrm{We}$ therefore suggest that there is no spatial separation of the generators for the rhythmic activities elicited by varied pain intensity within the SII areas.

In conclusion, power increases of 4-13 Hz oscillations peaking from 180 to $210 \mathrm{~ms}$ play an important role in processing A $\delta$ nociceptive inputs in the bilateral SII areas. Theta and alpha activities in the SII areas reflect the perceived pain magnitude up to a moderate degree, rather than a full scale, of pain rating. These rhythmic generators elicited by different pain intensity heavily overlapped within the SII areas.

\section{ACKNOWLEDGEMENTS AND FUNDING}

We thank Mr. Chih-Che Chou and Mr. Chou-Ming Cheng for their technical assistance in the acquisition of MR images. This study was supported in part by research grants from Taipei Veterans General Hospital (V96ER3-004; V96C1-123, V97ER3006, VGHUST97-PG24) and from the National Science Council (NSC-95-2314-B-010-030-MY3， NSC-96-2628-B010-030MY3, NSC-94-2811-B-010-009), Taipei, Taiwan.

\section{REFERENCES}

1. Mor J, Carmon A. Laser emitted radiant heat for pain research. Pain. 1975; 1: 233-7.

2. Bromm B, Jahnke MT, Treede RD. Responses of human cutaneous afferents to $\mathrm{CO}_{2}$ laser stimuli causing pain. Exp Brain Res. 1984; 55: $158-66$

3. Bromm B, Treede RD. Laser-evoked cerebral potentials in the assessment of cutaneous pain sensitivity in normal subjects and patients. Rev Neurol (Paris). 1991; 147: 625-43.

4. Kakigi R, Koyama S, Hoshiyama M, Kitamura Y, Shimojo M, Watanabe S. Pain-related magnetic fields following painful $\mathrm{CO}_{2}$ laser stimulation in man. Neurosci Lett. 1995; 192: 45-8.

5. Kakigi R, Koyama S, Hoshiyama M, Kitamura Y, Shimojo M, Watanabe $\mathrm{S}$. Pain-related brain responses following $\mathrm{CO}_{2}$ laser stimulation: magnetoencephalographic studies. Electroencephalogr Clin Neurophysiol Suppl. 1996; 47: 111-20.

6. Chen WT, Yuan RY, Shih YH, Yeh TC, Hung DL, Wu ZA, et al. Neuromagnetic SII responses do not fully reflect pain scale. Neuroimage. 2006; 31: 670-6.

7. Forss N, Raij TT, Seppa M, Hari R. Common cortical network for first and second pain. Neuroimage. 2005; 24: 132-42.

8. Timmermann L, Ploner M, Haucke K, Schmitz F, Baltissen R, Schnitzler A. Differential coding of pain intensity in the human primary and secondary somatosensory cortex. J Neurophysiol. 2001; 86: 1499-503.

9. Apkarian AV, Darbar A, Krauss BR, Gelnar PA, Szeverenyi NM. Differentiating cortical areas related to pain perception from stimulus identification: temporal analysis of fMRI activity. J Neurophysiol. 1999; 81: 2956-63.

10. Kwan CL, Crawley AP, Mikulis DJ, Davis KD. An fMRI study of the anterior cingulate cortex and surrounding medial wall activations evoked by noxious cutaneous heat and cold stimuli. Pain. 2000; 85: 359-74.

11. Peyron R, Garcia-Larrea L, Gregoire MC, Convers P, Richard A, Lavenne F, et al. Parietal and cingulate processes in central pain. A combined positron emission tomography (PET) and functional magnetic resonance imaging (fMRI) study of an unusual case. Pain. 2000; 84: 77-87.

12. Talbot JD, Marrett S, Evans AC, Meyer E, Bushnell MC, Duncan GH. Multiple representations of pain in human cerebral cortex. Science. 1991; 251: 1355-8.

13. Kakigi R, Inui K, Tamura Y. Electrophysiological studies on human pain perception. Clin Neurophysiol. 2005; 116: 743-63. 
14. Basar E. Brain function and oscillations. Integrative brain functions. Vol. I. Berlin: 1998.

15. Basar E. Brain function and oscillations. Integrative brain functions. Vol. II. Berlin: 1999

16. Basar E, Basar-Eroglu C, Karakas S, Schurmann M. Oscillatory brain theory: a new trend in neuroscience. IEEE Eng Med Biol Mag. 1999; 18: 56-66.

17. Basar E, Basar-Eroglu C, Karakas S, Schurmann M. Brain oscillations in perception and memory. Int $\mathrm{J}$ Psychophysiol. 2000; 35: 95-124.

18. Basar E, Ozgoren M, Karakas S, Basar-Eroglu C. Super-synergy in the brain: grandmother percept is manifested by multiple oscillations. Int J Bifurcat Chaos. 2004; 14: 1-38.

19. Klopp J, Halgren E, Marinkovic K, Nenov V. Face-selective spectral changes in the human fusiform gyrus. Clin Neurophysiol. 1999; 110: 676-82.

20. Quian Quiroga R, Sakowitz OW, Basar E, Schurmann M. Wavelet transform in the analysis of the frequency composition of evoked potentials. Brain Res Brain Res Protoc. 2001; 8: 16-24.

21. Yordanova J, Devrim M, Kolev V, Ademoglu A, Demiralp T. Multiple time-frequency components account for the complex functional reactivity of P300. Neuroreport. 2000; 11: 1097-103.

22. Yordanova J, Kolev V, Rosso OA, Schürmann M, Sakowitz OW, Ozgören M, et al. Wavelet entropy analysis of event-related potentials indicates modality-independent theta dominance. J Neurosci Methods. 2002; 117: 99-109.

23. Sarnthein J, Stern J, Aufenberg C, Rousson V, Jeanmonod D. Increased EEG power and slowed dominant frequency in patients with neurogenic pain. Brain. 2006; 129: 55-64.

24. Stern J, Jeanmonod D, Sarnthein J. Persistent EEG overactivation in the cortical pain matrix of neurogenic pain patients. Neuroimage. 2006; 31: 721-31.

25. Hauck M, Lorenz J, Engel AK. Attention to painful stimulation enhances gamma-band activity and synchronization in human sensorimotor cortex. J Neurosci. 2007; 27:9270-7.

26. Samar VJ, Swartz KP, Raghuveer MR. Multiresolution analysis of event-related potentials by wavelet decomposition. Brain Cogn. 1995; 27: 398-438.

27. Price DD, McGrath PA, Rafii A, Buckingham B. The validation of visual analogue scales as ratio scale measures for chronic and experimental pain. Pain. 1983; 17:45-56.

28. Gracely RH. Studies of pain in human subjects. In: Wall PD, Melzack R, editors. Textbook of pain, 4th ed. Edinburgh: Churchill Livingstone; 1999. p. 385-407.

29. Hämäläinen M, Hari R, Ilmoniemi RJ, Knuutila J, Lounasmaa OV. Magnetoencephalography - theory, instrumentation, and application to noninvasive studies of the working human brain. Rev Mod Phys. 1993; 65: 413-97.

30. Kronland-Martinet R, Morlet J, Grossmann A. Analysis of sound patterns through wavelet transforms. Int J Patt Recogn Art Intell. 1987; 1: 273-302.

31. Grossman A, Kronland-Martinet R, Morlet J. Reading and understanding continuous wavelets transforms. Wavelets, timefrequency methods and phase space. Berlin: Springer-Verlag; 1989. p. 2-20.

32. Hsiao FJ, Lin YY, Hsieh JC, Wu ZA, Ho LT, Chang Y. Oscillatory characteristics of face-evoked neuromagnetic responses. Int $\mathbf{J}$ Psychophysiol. 2006; 61: 113-20.

33. Jensen O, Gelfand J, Kounios J, Lisman JE. Oscillations in the alpha band $(9-12 \mathrm{~Hz})$ increase with memory load during retention in a short-term memory task. Cereb Cortex. 2002; 12 : $877-82$

34. Lachaux JP, Rodriguez E, Martinerie J, Varela FJ. Measuring phase synchrony in brain signals. Hum Brain Mapp. 1999; 8: 194-208.

35. Lin YY, Hsiao FJ, Shih YH, Yiu CH, Yen DJ, Kwan SY, et al. Plastic phase-locking and magnetic mismatch response to auditory deviants in temporal lobe epilepsy. Cereb Cortex. 2007; 17: 2516-25.

36. Rodriguez E, George N, Lachaux JP, Martinerie J, Renault B, Varela FJ. Perception's shadow: long-distance synchronization of human brain activity. Nature. 1999; 397: 430-3.
37. Tallon-Baudry C, Bertrand O, Delpuech C, Pernier J. Stimulus specificity of phase-locked and non-phase-locked $40 \mathrm{~Hz}$ visual responses in human. J Neurosci. 1996; 16: 4240-9.

38. Tallon-Baudry C, Bertrand O, Peronnet F, Pernier J. Induced gamma-band activity during the delay of a visual short-term memory task in humans. J Neurosci. 1998; 18: 4244-54.

39. Chen AC, Herrmann CS. Perception of pain coincides with the spatial expansion of electroencephalographic dynamics in human subjects. Neurosci Lett. 2001; 297: 183-6.

40. Bromm B, Meier W, Scharein E. Pre-stimulus/post-stimulus relations in EEG spectra and their modulations by an opioid and an antidepressant. Electroencephalogr Clin Neurophysiol. 1989; 73: 188-97.

41. Mouraux A, Guerit JM, Plaghki L. Non-phase locked electroencephalogram (EEG) responses to $\mathrm{CO}_{2}$ laser skin stimulations may reflect central interactions between A partial partial differential- and C-fibre afferent volleys. Clin Neurophysiol. 2003; 114: 710-22.

42. Mouraux A, Plaghki L. Single-trial detection of human brain responses evoked by laser activation of Adelta-nociceptors using the wavelet transform of EEG epochs. Neurosci Lett. 2004; 361 : 241-4.

43. Backonja M, Howland EW, Wang J, Smith J, Salinsky M, Cleeland CS. Tonic changes in alpha power during immersion of the hand in cold water. Electroencephalogr Clin Neurophysiol. 1991; 79: 192-203.

44. Chang PF, Arendt-Nielsen L, Graven-Nielsen T, Svensson P, Chen AC. Different EEG topographic effects of painful and nonpainful intramuscular stimulation in man. Exp Brain Res. 2001; 141: 195-203.

45. Chen AC, Dworkin SF, Haug J, Gehrig J. Topographic brain measures of human pain and pain responsivity. Pain. 1989; 37: $129-41$

46. Chen AC, Rappelsberger P. Brain and human pain: topographic EEG amplitude and coherence mapping. Brain Topogr. 1994; 7: $129-40$

47. Ferracuti S, Seri S, Mattia D, Cruccu G. Quantitative EEG modifications during the Cold Water Pressor Test: hemispheric and hand differences. Int J Psychophysiol. 1994; 17: 261-8.

48. Veerasarn P, Stohler CS. The effect of experimental muscle pain on the background electrical brain activity. Pain. 1992; 49: 349-60.

49. Babiloni C, Babiloni F, Carducci F, Cincotti F, Rosciarelli F, Arendt-Nielsen L, et al. Human brain oscillatory activity phaselocked to painful electrical stimulations: a multi-channel EEG study. Hum Brain Mapp. 2002; 15: 112-23.

50. Narici L, Forss N, Jousmaki V, Peresson M, Hari R. Evidence for a 7- to 9-Hz "sigma" rhythm in the human SII cortex. Neuroimage. 2001; 13: 662-8.

51. Bromm B, Chen AC. Brain electrical source analysis of laser evoked potentials in response to painful trigeminal nerve stimulation. Electroencephalogr Clin Neurophysiol. 1995; 95: 14-26.

52. Schlereth T, Baumgartner U, Magerl W, Stoeter P, Treede RD. Lefthemisphere dominance in early nociceptive processing in the human parasylvian cortex. Neuroimage. 2003; 20: 441-54.

53. Tarkka IM, Treede RD. Equivalent electrical source analysis of pain-related somatosensory evoked potentials elicited by a $\mathrm{CO}_{2}$ laser. J Clin Neurophysiol. 1993; 10: 513-9.

54. Valeriani M, Rambaud L, Mauguiere F. Scalp topography and dipolar source modelling of potentials evoked by $\mathrm{CO}_{2}$ laser stimulation of the hand. Electroencephalogr Clin Neurophysiol. 1996; 100: 343-53.

55. Nakamura Y, Paur R, Zimmermann R, Bromm B. Attentional modulation of human pain processing in the secondary somatosensory cortex: a magnetoencephalographic study. Neurosci Lett. 2002; 328: 29-32.

56. Raij TT, Vartiainen NV, Jousmaki V, Hari R. Effects of interstimulus interval on cortical responses to painful laser stimulation. J Clin Neurophysiol. 2003; 20: 73-9.

57. Watanabe S, Kakigi R, Koyama S, Hoshiyama M, Kaneoke Y. Pain processing traced by magnetoencephalography in the human brain. Brain Topogr. 1998; 10: 255-64. 
58. Coghill RC, Gilron I, Iadarola MJ. Hemispheric lateralization of somatosensory processing. J Neurophysiol. 2001; 85: 2602-12.

59. Coghill RC, Sang CN, Maisog JM, Iadarola MJ. Pain intensity processing within the human brain: a bilateral, distributed mechanism. J Neurophysiol. 1999; 82: 1934-43.

60. Knecht S, Kunesch E, Schnitzler A. Parallel and serial processing of haptic information in man: effects of parietal lesions on sensorimotor hand function. Neuropsychologia. 1996; 34: 669-87.

61. Stein BE, Price DD, Gazzaniga MS. Pain perception in a man with total corpus callosum transection. Pain. 1989; 38: 51-6.

62. Valeriani M, Restuccia D, Barba C, Le Pera D, Tonali P, Mauguiere F. Sources of cortical responses to painful $\mathrm{CO}_{2}$ laser skin stimulation of the hand and foot in the human brain. Clin Neurophysiol. 2000; 111: 1103-12.

63. Frot M, Garcia-Larrea L, Guenot M, Mauguiere F. Responses of the supra-sylvian (SII) cortex in humans to painful and innocuous stimuli. A study using intra-cerebral recordings. Pain. 2001; 94 : 65-73.

64. Frot M, Rambaud L, Guenot M, Mauguiere F. Intracortical recordings of early pain-related $\mathrm{CO}_{2}$-laser evoked potentials in the human second somatosensory (SII) area. Clin Neurophysiol. 1999; 110: 133-45.

65. Lenz FA, Rios M, Chau D, Krauss GL, Zirh TA, Lesser RP. Painful stimuli evoke potentials recorded from the parasylvian cortex in humans. J Neurophysiol. 1998; 80: 2077-88.
66. Peyron R, Frot M, Schneider F, Garcia-Larrea L, Mertens P, Barral FG, et al. Role of operculoinsular cortices in human pain processing: converging evidence from PET, fMRI, dipole modeling, and intracerebral recordings of evoked potentials. Neuroimage. 2002; 17: 1336-46.

67. Vogel H, Port JD, Lenz FA, Solaiyappan M, Krauss G, Treede RD. Dipole source analysis of laser-evoked subdural potentials recorded from parasylvian cortex in humans. J Neurophysiol. 2003; 89: 3051-60.

68. Bromm B, Lorenz J, Scharein E. Dipole source analysis of brain activity in the assessment of pain. Recent advances in clinical neurophysiology. Amsterdam: Elsevier; 1996. p. 328-35.

69. Kanda M, Nagamine T, Ikeda A, Ohara S, Kunieda T, Fujiwara N, et al. Primary somatosensory cortex is actively involved in pain processing in humans. Brain Res. 2000; 853: 282-9.

70. Ploner M, Schmitz F, Freund HJ, Schnitzler A. Parallel activation of primary and secondary somatosensory cortices in human pain processing. J Neurophysiol. 1999; 81: 3100-4. 